\title{
Total mass distributions of Sersic galaxies from photometry and central velocity dispersion
}

\author{
D. Chakrabarty ${ }^{1}$ and B. Jackson ${ }^{2}$
}

\author{
1 School of Physics \& Astronomy, University of Nottingham, Nottingham NG7 2RD, UK \\ e-mail: dalia.chakrabarty@nottingham.ac.uk \\ 2 Institute for Astronomy, University of Edinburgh, Blackford Hill, Edinburgh EH9 3HJ, UK \\ e-mail: bmj@roe.ac.uk
}

Received 14 April 2008 / Accepted 7 February 2009

\begin{abstract}
Aims. We develop a novel way of finding total mass density profiles in Sersic ellipticals, to about 3 times the major axis effective radius, using no other information other than what is typically available for distant galaxies, namely the observed surface brightness distribution and the central velocity dispersion $\sigma_{0}$.

Methods. The luminosity density profile of the observed galaxy is extracted by deprojecting the measured brightness distribution and scaling it by a fiduciary, step-function shaped, raw mass-to-light ratio profile $(M / L)$. The resulting raw, discontinuous, total, 3-D mass density profile is then smoothed according to a proposed smoothing prescription. The parameters of this raw $M / L$ are characterised by implementing the observables in a model-based study.

Results. The complete characterisation of the formalism is provided as a function of the measurements of the brightness distribution and $\sigma_{0}$. The formalism, thus specified, is demonstrated to yield the mass density profiles of a suite of test galaxies and is successfully applied to extract the gravitational mass distribution in NGC 3379 and NGC 4499, out to about 3 effective radii.
\end{abstract}

Key words. methods: data analysis - galaxies: fundamental parameters

\section{Introduction}

Any evaluation of the total mass in distant galaxies is a struggle against the paucity of available observational evidence. Photometry is hardly enough to indicate the content of both the luminous as well as the dark matter, unless the functional dependence between luminosity content and dark mass is accessible. This is of course not the case; the existence of such a relation is itself uncertain, especially in early type galaxies. While kinematic information of tracers has often been advanced as indicators of mass distributions in galaxies, the implementation of such information is tricky, primarily because of the mass-anisotropy degeneracy. Thus, one often resorts to cleverly designed observational techniques and/or algorithms and formalisms which thrive even in light of the limited measurements. Examples of these include the Planetary Nebula Spectrograph (Romanowsky et al. 2004), the NMAGIC code (de Lorenzi et al. 2007) and CHASSIS (Chakrabarty \& Saha 2001).

The X-ray emission from X-ray active systems can be analysed to offer insight into the gravitational mass distribution in the galaxy, under the assumption of hydrostatic equilibrium (Humphrey et al. 2008; Lemze et al. 2008; Mahdavi et al. 2008; Zhang et al. 2007; Fukazawa et al. 2006; O'Sullivan \& Ponman 2004, to cite some recent work). However, what makes this method potentially unreliable is the lack of information about the distribution of the fraction of hot gas that is in hydrostatic equilibrium (Churazov et al. 2008; Diehl \& Statler 2007).

Comparatively, a more stable route to mass distribution determination is via lensing measurements. However, the biggest shortcoming of mass determination from lensing measurements alone is the unavailability of the full three-dimensional mass distribution. To improve upon this, lensing data is often supplemented by dynamically obtained mass estimates (Czoske et al. 2008; Bolton et al. 2008; Gavazzi et al. 2007; Koopmans \& Treu 2003).

However, there are questionable implementational problems involved in (parametric) dynamical mass determination, the chief of which are typically the mass-anisotropy degeneracy, binning-triggered instability of scant velocity dispersion data, reliance on the modelling of the stellar mass density and an even more fundamental worry caused by the assumption of one smooth parametric representation of the phase-space distribution function of the used tracer and by its relation to the phase-space density of the whole galaxy. This is of course in addition to the uncertainties in the mass distribution resulting from substituting the real geometry of the system by sphericity, as is typically done with all mass determination procedures. Above all, using tracer kinematics for mass determination (de Lorenzi et al. 2008; Douglas et al. 2007, etc.) is limited in applicability given the reliance on the size of the tracer kinematic data! Large data sets are of course hard to attain in systems that are not close by. Moreover, this method is unsuitable for fast evaluation of the mass distribution of individual galaxies that are members of a large sample, as for example, a galaxy obseved in a large survey.

On the contrary, it would be highly beneficial to design a method that is comparatively less data-intensive in that it demands only what is easily available from observations. We advance a methodology that provides total gravitational mass density distributions to about 3 effective radii, as compared to only 1 effective radius (Cappellari et al. 2006), in a fast and easy-toimplement fashion.

This advanced formalism is inspired by a trick that was reported in Chakrabarty (2007, hereafter, Paper I). This trick 
involves the exploitation of only photometry and the central velocity dispersion measure $\left(\sigma_{0}\right)$ in a galaxy, in order to generate the total local mass-to-light ratio $(M / L)$ profile to a distance that is about thrice the semi-major axis effective radius. This cutoff distance is described in details below. The prescription for constructing this profile was provided in Paper I, though only for a certain class of power-law galaxies. However, the exact nature of this prescription is very much a function of the photometric class that the galaxy belongs to. Thus, the formula reported in Paper I cannot be invoked to shed light on the mass distribution in galaxies that betray a different (and more ubiquitous) photometric class, eg. ellipticals, the surface brightness of which can be fit by a Sersic profile (Sersic 1968). This is precisely what is reported in this paper.

This paper is arranged as follows: the basic framework of the suggested formalism is discussed in Sect. 2, followed by a note on the models that we use. The method used to obtain the sought functional forms is briefly mentioned in Sect. 4. Results obtained from our work are subsequently discussed in Sect. 5. Tests of the method are described in Sect. 6 while Sect. 7 deals with applications to real galaxies NGC 3379 and NGC 4494. The paper is rounded off with a section devoted to discussions of relevant points.

\section{Formalism}

The only Sersic model that was considered in Paper I did actually indicate that the mass estimation trick suggested for the powerlaw systems might be possible for Sersic galaxies too. Following this lead, as in Paper I, we first invoke a raw two-stepped $M / L$ profile of the Sersic galaxy at hand, where it is the distribution of $M / L$ with the major axis coordinate $x$, that is relevant. This raw $M / L$ distribution is subsequently smoothed, (according to the smoothing prescription provided in Paper I and discussed below) to provide the real $M / L$ distribution of the system, to a distance that is by definition, 3 times the major-axis effective radii for a Sersic galaxy with sersic index $n=4$ but is an approximation for the major-axis effective radius for all other values of $n$. The formal definition of this distance is given in Eq. (1) while the justification for our choice of this length scale is delineated in Sect. 8.4. Figure 1 represents a schematic diagram of this raw $M / L$ profile against $x$.

As can be appreciated from Fig. 1, the unprocessed $M / L$ profile is a two-stepped function that is characterised by three free parameters, including the position of the step, or the jump radius $x_{\text {in }}$ and the $M / L$ amplitudes inside and outside $x_{\text {in }}$. The object of the current exercise is to fully characterise this unprocessed $M / L$ profile, so that upon smoothing, the final, total, local $M / L$ distribution over $x$ is retrieved. This final form of the $M / L$ distribution is significantly different from the discontinuous, 2-stepped, raw $M / L$ that we initially choose to work with. In fact, the final form of the profile is smooth and the $M / L$ values vary a lot with $x$, sometimes abruptly, depending on the details of the model galaxy. Figure 4 in Paper I represents a comparison of one such raw $M / L$ profile and the final smoothed $M / L$ distribution that is advanced as the representative $M / L$ profile for the test system at hand. In fact, later in Fig. 6, a raw $M / L$ (exemplified in Fig. 1) is compared to the true $M / L$ distribution of the test galaxy under consideration - the difference between the raw and final forms of the $M / L$ profiles is clear in that figure.

As in Paper I, $x_{\mathrm{in}}$ is set equal to $3 X_{\mathrm{e}}$, where

$X_{\mathrm{e}}=\left(\frac{-3.33}{m}\right)^{4}$

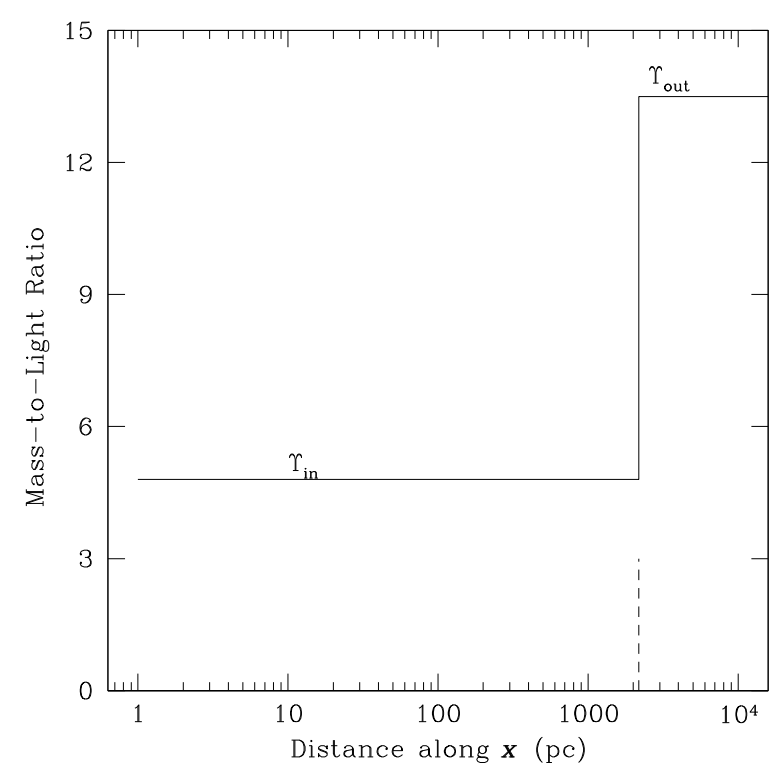

Fig. 1. Schematic view of a raw $M / L$ distribution along the major axis coordinate $x$, for a model galaxy. The dashed line marks the position of the jump radius $x_{\text {in }}$ which as defined in Eq. (1), is 3 times the major axis effective radius of the model galaxy, if the galaxy is described by a Sersic index of 4; otherwise, $x_{\mathrm{in}}$ is an approximation for the majoraxis effective radius. The amplitudes inside and outside $X_{\text {in }}$ have been marked as $\Upsilon_{\text {in }}$ and $\Upsilon_{\text {out }}$. While the measured central velocity dispersion is used to estimate the range of $\Upsilon_{\text {in }}$ values allowed, $\Upsilon_{\text {out }}$ is constrained via the sought analytical relation that connects $\Upsilon_{\text {out }}$ to $\Upsilon_{\text {in }}$. The point of this paper is to seek such relations, in order to fully characterise the raw $M / L$ profile; once the raw $M / L$ distribution is known, it is smoothed to obtain the final, total $M / L$ ratio profile of the galaxy, which is significantly different from this unprocessed $M / L$ shown above.

and $m$ is the slope of the straight line that is fit to the plot of core-removed $\log _{10}(I)$ against $x^{1 / 4}$ (as in Paper I), i.e. $X_{\mathrm{e}}$ is the major axis equivalent of the effective radius, if the Sersic index is 4 . For all other values of the Sersic index, $X_{\mathrm{e}}$ is at most an approximation to the major axis effective radius. The advantage of using $X_{\mathrm{e}}$ over the exact definition of the major-axis effective radius is described below in Sect. 8.4.

The smoothing prescription used here is the same as in Paper I - we smooth the raw total mass density profile by two successive applications of a box filter of size corresponding to $X_{\mathrm{e}}$.

The amplitude of $M / L(x)$ for $x \leq x_{\text {in }}$ is referred to as $\Upsilon_{\text {in }}$ and that for $x>x_{\text {in }}$ it is $\Upsilon_{\text {out }}$. Now that $x_{\text {in }}$ has been pinned down by construction, we hope to get a constraint on the available choices for $\Upsilon_{\text {in }}$ from the central velocity dispersion $\left(\sigma_{0}\right)$ and also hope to identify a functional dependence of $\Upsilon_{\text {out }}$ on $\Upsilon_{\text {in }}$. The exact form of such a constraint or function is yet unknown but we begin by expecting these to be defined in terms of the photometric parameters that describe the surface brightness profile of the Sersic galaxy under consideration, namely, the Sersic index $n$ and $X_{\mathrm{e}}$ that we have defined above (Eq. (1)). The central brightness is not a free parameter since we normalise all luminosity density profiles to a central value of $1000 L_{\odot} \mathrm{pc}^{-3}$. Thus, we want to find the function $f\left(n, X_{\mathrm{e}}, \Upsilon_{\text {in }}\right)$, where

$\Upsilon_{\text {out }}=f\left(n, X_{\mathrm{e}}, \Upsilon_{\mathrm{in}}\right)$

The inspiration for the hypothesis that $f$ depends on the photometric parameters is discussed below. 
Given that by construction, $\Upsilon_{\text {in }}$ is the uniform amplitude of the raw $M / L$ profile for $x<x_{\text {in }}$, we expect it to be related to the "central" mass-to-light ratio of the galaxy, where by "central" is implied the distance at which the measurement of the central velocity dispersion $\left(\sigma_{0}\right)$ is obtained (at $x=x_{0}$, with $x_{0}$ typically less than $x_{\text {in }}$ ). However, in a real system, the true central $M / L$ cannot be securely determined from the measurement of $\sigma_{0}$ alone, owing to uncertainties about the validity of the assumptions that are invoked, in order to translate knowledge of $\sigma_{0}$ to that of mass enclosed within $x_{0}$ (using virial theorem). Such uncertainties basically stem from the presence of anisotropy in phase-space. In the mass modelling trick advanced in Paper I, room is allowed for the accommodation of such uncertainties, as long as the deviations from the assumptions used in the virial estimate of mass are not atypically more than what has been observed with real ellipticals (Padmanabhan et al. 2004); this is discussed in the following paragraph.

In Paper I, the virial estimate of the central $M / L$, from $\sigma_{0}$ was parametrised by $\alpha$. It was found that for a measured $\sigma_{0}$, as long as $\Upsilon_{\text {in }}$ lies within a range of values (the details of this range correspond to the given $\alpha$ ), compatibility between the predicted and known (model) mass density distributions is ensured. In other words, for $\alpha$ calculated from a given $\sigma_{0}, \Upsilon_{\text {in }}$ can be safely chosen to belong to a range of $M / L$ values: $\Upsilon_{\text {in }}^{\min }$ to $\Upsilon_{\text {out }}^{\text {max }}$. Such positioning of $\Upsilon_{\text {in }}$ can be checked by comparing the mass distributions recovered with $\Upsilon_{\text {in }}=\Upsilon_{\text {in }}^{\text {min }}$ and $\Upsilon_{\text {in }}=\Upsilon_{\text {in }}^{\max }$, for consistency. Here $\Upsilon_{\text {in }}^{\min }=\alpha$ while $\Upsilon_{\text {in }}^{\max }$ is an unknown function of the photometric parameters and $\alpha$ - say $g\left(n, X_{\mathrm{e}}, \alpha\right)$,

$\Upsilon_{\mathrm{in}}^{\max }=g\left(n, X_{\mathrm{e}}, \alpha\right)$

where $g\left(n, X_{\mathrm{e}}, \alpha\right)$ is unknown and the choice of its dependence on the photometric parameters is the following. In Paper I we had success upon choosing the functions $f$ and $g$ as dependent on the photometric details of the system; this was the case for a suite of model power-law galaxies. Such "success" is qualified in terms of the identification of the hypothesised dependence on the photometric properties of the model galaxies. Motivated by this, we endeavour to find forms of $f$ and $g$.

Thus, there are two unknown functions that we wish to constrain: $f\left(n, X_{\mathrm{e}}, \Upsilon_{\mathrm{in}}\right)$ and $g\left(n, X_{\mathrm{e}}, \alpha\right)$. These functions, when known, will provide $\Upsilon_{\text {out }}$ from $\Upsilon_{\text {in }}$ which will be known from $\alpha$, (i.e. $\left.\sigma_{0}\right)$ and the observed brightness distribution of the galaxy. Once we know $\Upsilon_{\text {out }}$ from $\Upsilon_{\text {in }}$, we would then have fully characterised the raw $M / L$ distribution over $x$. The smoothed out version of this raw $M / L$ profile will then be advanced as the true $M / L$ profile of the system at hand. This in conjunction with the luminosity density distribution will allow knowledge of the mass density distribution.

We hope to recover the analytical forms of these two unknown functions for Sersic galaxies to $3 X_{\mathrm{e}}$, through an analysis of a sample of model Sersic galaxy surface brightness profiles. Analytical fits are sought to the data that is collated from the suite of models that we work with, in order to recognise patterns, if any, that may show up in the relations between the various quantities, in particular, $\Upsilon_{\text {out }}-\Upsilon_{\text {in }}$ and $\Upsilon_{\text {in }}-\alpha$. The variation in these relations with changing models is then explored to unravel the reliance of these relations on the photometric parameters.

\section{Models}

We identify the relation between $\Upsilon_{\text {in }}$ and $\Upsilon_{\text {out }}$ in Sersic galaxies, by recognising (and then quantifying) the patterns that emerge between these quantities, as diverse model galaxies are scanned.
To reminisce, Sersic galaxies are those, the surface brightness profile $I(R)$ of which can be approximated as:

$I(R)=I_{0} \exp \left[-b_{n}\left(R / R_{\mathrm{e}}\right)^{1 / n}\right]$

where $I_{0}$ is the central surface brightness, $R_{\mathrm{e}}$ is the projected effective half-light radius, $n$ is the Sersic index that determines the curvature of the brightness profile and $b_{n}$ is a function of $n$ : for $n>0.5, b_{n} \approx 2 n-1 / 3+0.009876 / n$ (Prugniel \& Simien 1997; Lima Neto et al. 1999). Thus, the Sersic model represents a 3-parameter family. The corresponding deprojected spherical luminosity density distribution is discussed in Terzić \& Graham (2005); Mazure \& Capelato (2002). In Terzić \& Sprague (2007), the spherical models of Terzić \& Graham (2005) were generalised to include triaxiality. The luminosity density profile in the triaxial case is approximately given as:

$$
\begin{aligned}
\rho_{\mathrm{L}}(s) & =\rho_{0}\left(\frac{R_{\mathrm{e}}}{s}\right)^{p} \mathrm{e}^{-b_{n}\left(s / R_{\mathrm{e}}\right)^{1 / n}} \quad \text { where } \\
p & =1.0-\frac{0.6097}{n}+\frac{0.05563}{n^{2}} \text { for } n \in(0.6,10) .
\end{aligned}
$$

Here, the equation for $p$ is due to Lima Neto et al. (1999) and the ellipsoidal coordinate $s$ is defined as

$s^{2} \equiv \frac{x^{2}}{a^{2}}+\frac{y^{2}}{b^{2}}+\frac{z^{2}}{c^{2}}$,

for the axial ratios of a:b:c for the ellipsoidal system at hand. We use $X_{\mathrm{e}}$ in place of $R_{\mathrm{e}}$ (see Sect. 7.5). It is to be noted that Eq. (5) is an approximation and other forms have been used by Trujillo et al. (2002).

As in Paper I, in our models, we assume oblateness and an inclination of $90^{\circ}$, with a uniform projected axial ratio of 0.7 ; the work of Padilla \& Strauss (2008) corroborates such considerations of geometry and ellipticity. Also, the model systems are assumed to be viewed in the ACS $z$-band, at a distance of $17 \mathrm{Mpc}$. Though we recover the sought functions for these chosen configurations, generalisations to other systems will be suggested in Sects. 5.3 and 5.4.

Actually Terzić \& Sprague (2007) gives the more general 5-parameter mass density model which describes Sersic galaxies with a core inside a given break radius. However, we work with the simpler case of Sersic galaxies that can be qualified by a 3-parameter model. In fact, we constrict this further, by normalising the central luminosity density $\rho_{0}$ to $1000 M_{\odot} \mathrm{pc}^{-3}$. Also, we look for the luminosity distribution along the major axis. Thus, our models are distinguished only by the Sersic index $n$ and the $X_{\mathrm{e}}$ defined in Eq. ( 1).

Models were formulated for Sersic indices $n=3,4,5.3,6$, 7, 8 and 9 with $X_{\mathrm{e}}$ of $726 \mathrm{pc}, 1000 \mathrm{pc}, 1400 \mathrm{pc}, 2000 \mathrm{pc}$. While these models were extensively explored to achieve the sought functions, we investigated selected models with $n$ lying in this range and higher $X_{\mathrm{e}}$ values. In particular, we are interested in models with Sersic index $\leq 4$, with larger $X_{\mathrm{e}}$. Our viable models include galaxies with $n=3, X_{\mathrm{e}} \leq 4 \mathrm{kpc}$ and $n=4, X_{\mathrm{e}} \leq 5 \mathrm{kpc}$. Such an extended size range holds special interest for the greater fraction of observed ellipticals that correspond to this low $n$. Additionally, models with other $n$ were also found to work to $X_{\mathrm{e}}=10 \mathrm{kpc}$. This choice of models is supported by the results that

- In the nearby Virgo cluster, the ACSVCS survey (Ferrarese et al. 2006) reports that nearly $90 \%$ of the targetted earlytype systems (Sersic and cored-Sersic included) fall in the 

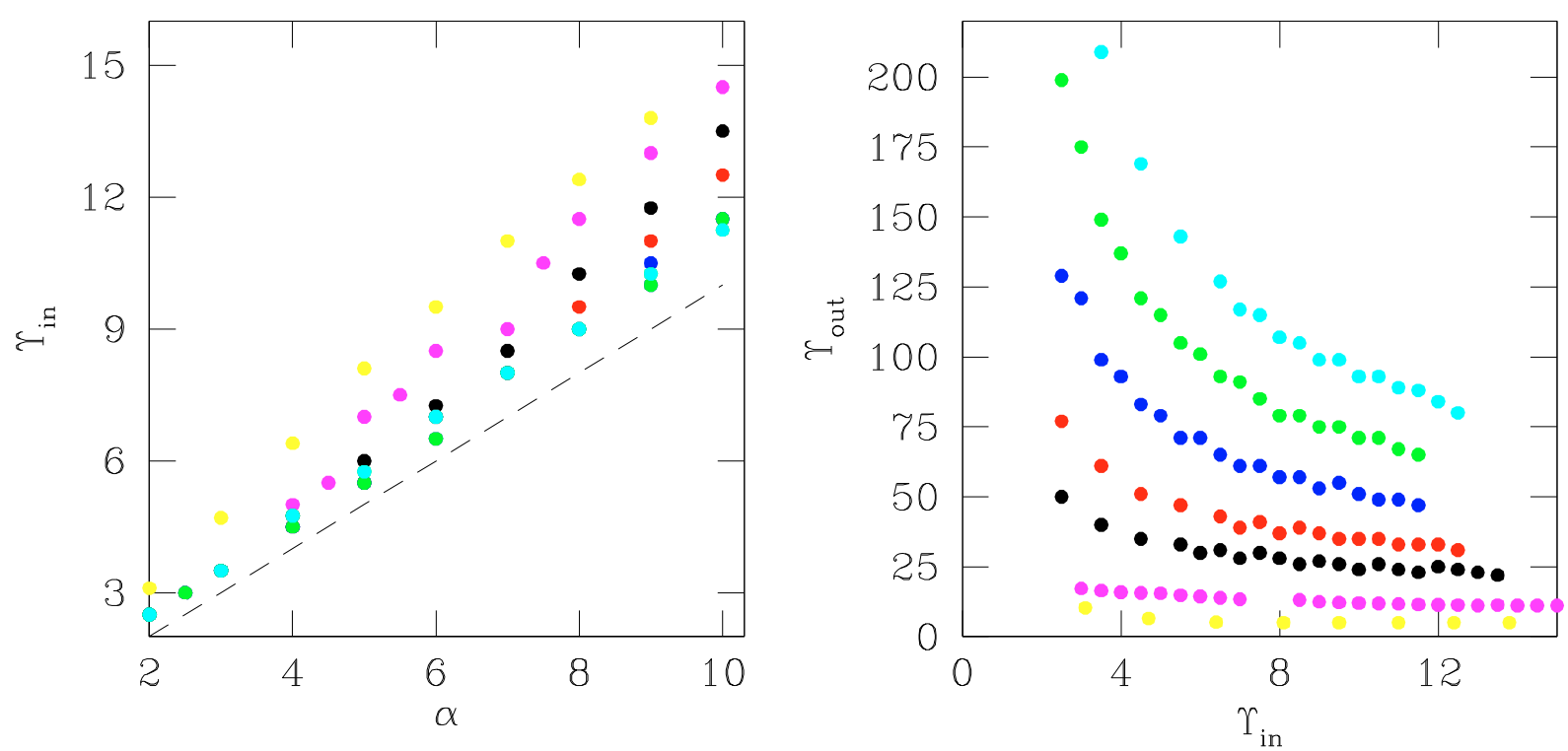

Fig. 2. Right panel: trends in $\Upsilon_{\text {out }}$ with $\Upsilon_{\text {in }}$, with changing Sersic index $n$, from model galaxies with $X_{\mathrm{e}}=726$ pc. The colour coding used for the different $n$ values is: yellow for $n=3$, magenta for $n=4$, black for $n=5.3$, red for $n=6$, blue for $n=7$, green for $n=8$ and cyan for $n=9$. In the left panel, $\Upsilon_{\mathrm{in}}^{\min }$ is plotted against $\alpha$ in broken lines; this plot is universally given by $\Upsilon_{\mathrm{in}}^{\min }=\alpha . \Upsilon_{\mathrm{in}}^{\max }$ is plotted against $\alpha$ for the different model galaxies used for the figure on the right, with the same colour coding. For a model at hand, at a given $\alpha, \Upsilon_{\text {in }}$ can be chosen from the range defined by the values of $\Upsilon_{\text {in }}^{\min }$ and $\Upsilon_{\text {in }}^{\max }$.

range of $R_{\mathrm{e}} \lesssim 5 \mathrm{kpc}$ in the $z$-band (which is the waveband directly comparable to our models). All the programme galaxies with $n \in[3,4]$ were found to have $R_{\mathrm{e}} \leq 2.2 \mathrm{kpc}$ while the single galaxy with $n \in(4,5.3]$ and $R_{\mathrm{e}}>5 \mathrm{kpc}$, is not a Sersic galaxy but is a cored Sersic system. Here, we remind ourselves that the reported effective radius $R_{\mathrm{e}}$ is really the geometric mean of the extent along the photometric semiaxes. Thus, the extent along the semi-major axis is greater (by about a factor of about 1.2, for an axial ratio of 0.7 ) than the reported $R_{\mathrm{e}}$. In other words, the surrogate for $X_{\mathrm{e}}$ is about 1.2 times the values of $R_{\mathrm{e}}$ quoted earlier in this paragraph. Even when this factor in taken into account, the range of our models covers the programme galaxies of the ACSVCS.

- Measurement of effective radius is waveband dependent (Temi et al. 2008; Ko \& Im 2005), so that for $n=3$ and 4 , our limits on the model $X_{\mathrm{e}}$ values in the $z$-band, are compatible with observations (Ferrarese et al. 2006; Trujillo et al. 2001; La Barbera et al. 2005).

- At high redshifts, systems display evolution towards higher compactness (Buitrago et al. 2008). Though this evolution is marked for systems at $z>1.7$ (sample of Buitrago et al. 2008), Ferreras et al. (2009) suggest a milder size evolution for the most massive galaxies for $0.4 \leq z \leq 1.2$. Thus, in the higher redshift range that we propose our scheme to be most useful in, if anything, the size range of our models would be more suitable.

This corroborates our choice of models, particularly in regard to the values of $X_{\mathrm{e}}$.

Sersic indices 2 or lower appeared not to be viable for the formalism to function, implying that this formalism is suitable only for elliptical systems (see Sect. 3). Sersic indices greater than 9 were not examined since such systems are very rare.

These luminosity density models, described by Eq. (5) and the used values of $n$ and $X_{\mathrm{e}}$, were embedded in an NFW-type dark halo (Navarro et al. 1996) of mass density $\rho_{\text {dark }}$, to give a total mass density of:

$\rho_{\mathrm{t}}(x)=\rho_{\mathrm{dark}}(x)+\alpha \rho_{\mathrm{L}}(x)$, where by choice,

$\rho_{\mathrm{dark}}(x)=\frac{M_{\mathrm{s}}}{4 \pi x\left(x+r_{\mathrm{s}}\right)^{2}}$.

Here $M_{\mathrm{s}}$ and $r_{\mathrm{s}}$ are mass and length scales of the halo, respectively. Our results are valid for halo parameters that correspond to the points in the green quadrilateral in the $M_{\mathrm{S}}-r_{\mathrm{S}}$ space that is depicted in the right panel of Fig. 10.

We ascribe $\pm 1-\sigma$ errors of about $10 \%$ to the luminosity density distributions that we generate and search for compatibility between the model mass density distribution and the predicted one, within these error bars.

\section{Method}

Combinations of the parameters $\alpha, \Upsilon_{\text {in }}$ and $\Upsilon_{\text {out }}$ that showed compatability with the known (model) mass density profiles, up to $3 X_{\mathrm{e}}$, at the aforementioned four separate $\left(M_{\mathrm{s}}, r_{\mathrm{s}}\right)$ coordinates, were searched for by our smoothing formalism that is automated.

We record the list of $\Upsilon_{\text {in }}$ values that imply compatibility for a chosen $\alpha$ (chosen typically in the range of 2 to 10). The starting value of $\Upsilon_{\text {in }}$ was typically about $\alpha$ while the upper value was set as $3 \alpha$, which was always sufficient to find the whole range of $\Upsilon_{\text {in }}$ values that correspond to compatability. Similarly, we record the $\Upsilon_{\text {out }}$ value corresponding to a given $\Upsilon_{\text {in }}$.

The plots of $\Upsilon_{\text {in }}^{\min }$ and $\Upsilon_{\text {in }}^{\max }$, as functions of $\alpha$ are monitored, with the aim of recognising the analytical form of these functional dependences. Similarly, the plot of $\Upsilon_{\text {out }}$ as a function of $\Upsilon_{\text {in }}$ is analysed at different $n$ and $X_{\mathrm{e}}$, to identify $f\left(n, X_{\mathrm{e}}, \Upsilon_{\text {in }}\right)$.

\section{Results}

With $X_{\mathrm{e}}$ held at $726 \mathrm{pc}$, as $n$ is varied, the plots of $\Upsilon_{\text {in }}$ against $\alpha$ are shown in the left panel of Fig. 2 while the relation between $\Upsilon_{\text {out }}$ against $\Upsilon_{\text {in }}$ is depicted for these models, in the right panel of this figure. 


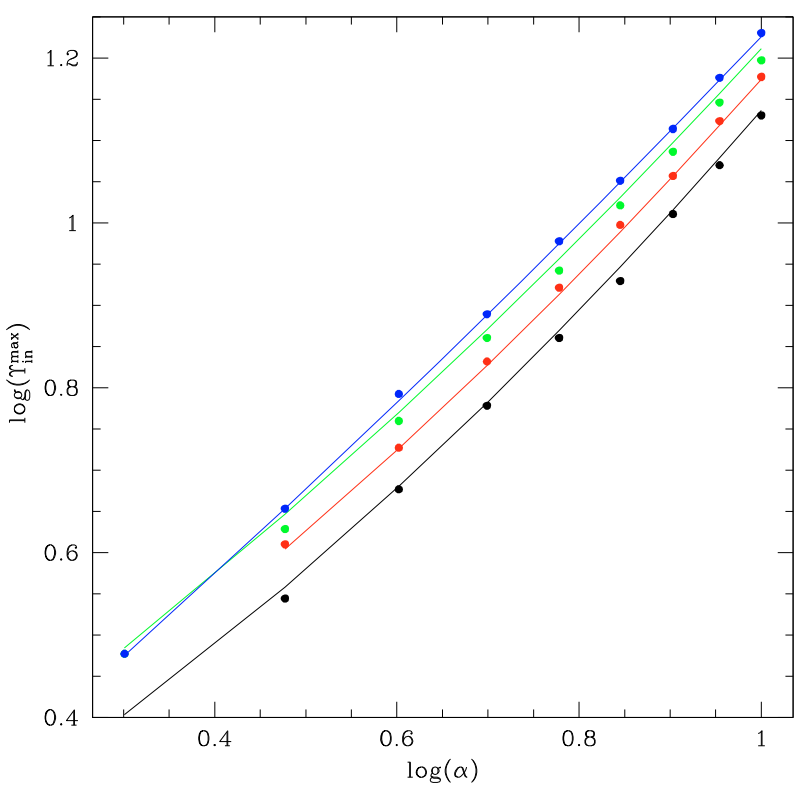

Fig. 3. The values of $\log \Upsilon_{\text {max }}^{\max }$ obtained at distinct values of $\log \alpha$, from our models, for $n=5.3$ and different $X_{\mathrm{e}}$; in black filled circles for $X_{\mathrm{e}}=$ $726 \mathrm{pc}$, red for $X_{\mathrm{e}}=1000 \mathrm{pc}$, green for $X_{\mathrm{e}}=1400 \mathrm{pc}$ and blue for $X_{\mathrm{e}}=2000$ pc. The predicted dependence of $\log \Upsilon_{\mathrm{in}}^{\max }$ on $\log \alpha$ (Eq. (9)) is plotted in solid lines, for the 4 different values of $X_{\mathrm{e}}$ that we use, in corresponding colours. This functional form holds approximately for other values of $n$ as well $(n \geq 3)$; the amplitude of $\Upsilon_{\text {in }}^{\max }$ only increases slightly with $n$.

\subsection{Relation between $\Upsilon_{\text {in }}$ and $\alpha$}

When the data collated from the different models is plotted it is noted that while $\Upsilon_{\text {in }}^{\min }=\alpha$, the $\log \left(\Upsilon_{\text {in }}^{\max }\right)-\log (\alpha)$ relation is well fit by a quadratic function of $\log (\alpha)$. This latter functional form has only a weak bearing on the Sersic index $n$, though $\Upsilon_{\text {in }}^{\max }$ is found to increase slightly as $n$ is increased from 5.3 to 9 . Thus, by denying this increased amplitude of $\log \Upsilon_{\text {in }}^{\max }$ with higher values of $n$, we merely constrict the range of values from which $\Upsilon_{\text {in }}$ can be chosen. Thus, our attempt at simplification of the sought functional form preempts a small reduction in the applicability of our formalism to more anisotropic galaxies than what is effectively allowed.

In Fig. 3, we present the values of $\log \left(\Upsilon_{\text {in }}^{\max }\right)$ at distinct values of $\log (\alpha)$, for different $X_{\mathrm{e}}$, and a single Sersic index of 5.3. The functional form of the relation is recovered from our analysis, and this is over-plotted on the data, in solid lines. This functional form is given by:

$$
\begin{aligned}
\Upsilon_{\mathrm{in}}^{\max }= & A_{0}\left(X_{\mathrm{e}}\right)+A_{1}\left(X_{\mathrm{e}}\right) \log \alpha+A_{2}\left(X_{\mathrm{e}}\right)(\log \alpha)^{2} \\
A_{0}\left(X_{\mathrm{e}}\right)= & -0.00747849+0.000360389 X_{\mathrm{e}} \\
& -1.31416 \times 10^{-7} X_{\mathrm{e}}^{2} \\
A_{1}\left(X_{\mathrm{e}}\right)= & 0.597653-2.61661 \times 10^{-5} X_{\mathrm{e}} \\
& +9.34449 \times 10^{-8} X_{\mathrm{e}}^{2} \\
A_{2}\left(X_{\mathrm{e}}\right)= & 0.396038-7.77987 \times 10^{-5} X_{\mathrm{e}} \\
& -3.03313 \times 10^{-8} X_{\mathrm{e}}^{2} .
\end{aligned}
$$

\subsection{Relation between $\Upsilon_{\text {out }}$ and $\Upsilon_{\text {in }}$}

The data from the assorted models indicate that $\Upsilon_{\text {out }}$ is noted to fall exponentially with $\Upsilon_{\text {in }}$; in fact, a good fit to this relation is given by the following equation:

$\Upsilon_{\text {out }}=\Delta\left(n, X_{\mathrm{e}}\right)+A_{0}\left(n, X_{\mathrm{e}}\right) \exp \left[-\Upsilon_{\text {in }} / \tau\left(n, X_{\mathrm{e}}\right)\right]$, where $\Delta, A_{0}$ and $\tau$ are functions of $X_{\mathrm{e}}$ and $n$. The relations between $n$ and $\Delta, A_{0}$ and $\tau$ are shown in the left, middle and right panels of Fig. 4, for the four different $X_{\mathrm{e}}$ values that we use. From our models, we seek the functional form of these relations; these recovered functions are over-plotted in solid lines, on the data in the three panels in Fig. 4. This comparison indicates that the known trends in $\log \Delta, \ln A_{0}$ and $\tau(n)$, with increasing $\log (n)$ and $n$, for the assorted $X_{\mathrm{e}}$ values, are well replicated by the predicted functional forms of the relevant quantities.

We advance the following formulae for $\Delta, A_{0}$ and $\tau$ :

$$
\begin{aligned}
\log \left[\Delta\left(n, X_{\mathrm{e}}\right)\right]= & C_{0}\left(X_{\mathrm{e}}\right)+C_{1}\left(X_{\mathrm{e}}\right) \log (n) \text { where } \\
C_{0}\left(X_{\mathrm{e}}\right)= & 0.18832-8.40018 \times 10^{-4} X_{\mathrm{e}} \\
& -1.19449 \times 10^{-7} X_{\mathrm{e}}^{2} \text { and } \\
C_{1}\left(X_{\mathrm{e}}\right)= & 1.15563+0.00207 X_{\mathrm{e}}-2.06978 \times 10^{-7} X_{\mathrm{e}}^{2} \\
\ln \left[A_{0}\left(n, X_{\mathrm{e}}\right)\right]= & D_{0}\left(X_{\mathrm{e}}\right)+D_{1}\left(X_{\mathrm{e}}\right) n+D_{2}\left(X_{\mathrm{e}}\right) n^{2} \\
& +D_{3}\left(X_{\mathrm{e}}\right) n^{3} \text { where } \\
D_{0}\left(X_{\mathrm{e}}\right)= & 480.078-1.32685 X_{\mathrm{e}}+0.00106312 X_{\mathrm{e}}^{2} \\
& -2.63527 \times 10^{-7} X_{\mathrm{e}}^{3}, \\
D_{1}\left(X_{\mathrm{e}}\right)= & -192.617+0.537814 X_{\mathrm{e}}-0.000431932 X_{\mathrm{e}}^{2} \\
& +1.07350 \times 10^{-7} X_{\mathrm{e}}^{3}, \\
D_{2}\left(X_{\mathrm{e}}\right)= & 24.9361-0.0699530 X_{\mathrm{e}}+5.63996 \times 10^{-5} X_{\mathrm{e}}^{2} \\
& -1.40766 \times 10^{-8} X_{\mathrm{e}}^{3} \text { and } \\
D_{3}\left(X_{\mathrm{e}}\right)= & -1.03518+0.00293420 X_{\mathrm{e}}-2.37672 \times 10^{-6} X_{\mathrm{e}}^{2} \\
& +5.96666 \times 10^{-10} X_{\mathrm{e}}^{3} .
\end{aligned}
$$

$$
\begin{aligned}
\tau\left(n, X_{\mathrm{e}}\right)= & \tau_{0}\left(X_{\mathrm{e}}\right)+\tau_{1}\left(X_{\mathrm{e}}\right) n+\tau_{2}\left(X_{\mathrm{e}}\right) n^{2} \\
& +\tau_{3}\left(X_{\mathrm{e}}\right) n^{3}+\tau_{4}\left(X_{\mathrm{e}}\right) n^{4} \quad \text { where } \\
\tau_{0}\left(X_{\mathrm{e}}\right)= & -3650.81+8.99277 X_{\mathrm{e}}-0.00649587 X_{\mathrm{e}}^{2} \\
& +1.43425 \times 10^{-6} X_{\mathrm{e}}^{3} \\
\tau_{1}\left(X_{\mathrm{e}}\right)= & 1903.74-4.64150 X_{\mathrm{e}}+0.00331538 X_{\mathrm{e}}^{2} \\
& -7.22203 \times 10^{-7} X_{\mathrm{e}}^{3} \\
\tau_{3}\left(X_{\mathrm{e}}\right)= & -367.254+0.885327 X_{\mathrm{e}}-0.000623864 X_{\mathrm{e}}^{2} \\
& +1.33667 \times 10^{-7} X_{\mathrm{e}}^{3} \text { and } \\
\tau_{4}\left(X_{\mathrm{e}}\right)= & 31.1895-0.0742165 X_{\mathrm{e}}+5.14858 \times 10^{-5} X_{\mathrm{e}}^{2} \\
& -1.08171 \times 10^{-8} X_{\mathrm{e}}^{3}
\end{aligned}
$$

These relations are valid for all model systems with $n \geq 3$ and that too, for $n=3$, only systems with $X_{\mathrm{e}} \leq 4000 \mathrm{pc}$ and for $n=4, X_{\mathrm{e}} \leq 5000 \mathrm{pc}$.

\subsection{Photometry in other wave-bands}

It is always possible that the observed photometry is presented in a wave-band different from the ACS $z$-band for which we predict the above relations between the properties of the raw $M / L$, though the $z$-band is in general a better choice for high redshift systems than a bluer waveband. If the available photometry is in a band different from $z$, (say the $w$-band), then the factor by which every value of $\Upsilon_{\text {in }}$ and $\Upsilon_{\text {out }}$ should be changed, is obtained from the following considerations. We realise that if $w$ is such that a model galaxy is brighter in $z$ than in the $w$-band, then the $M / L$ ratio by which the inner and outer parts of the brightness profile in $z$ need to be scaled, are smaller than the same by which 

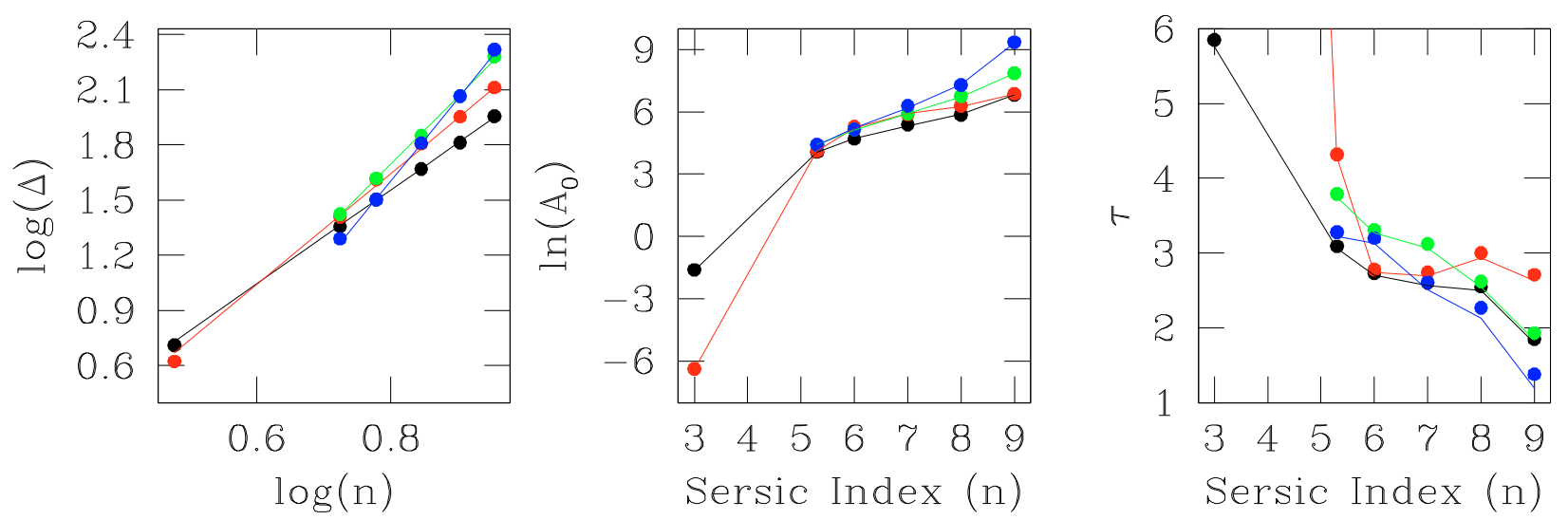

Fig. 4. Trends in the three different parameters that define the exponential fall-off of $\Upsilon_{\text {out }}$ with $\Upsilon_{\text {in }}$, with changes in $n$ and $X_{\mathrm{e}}$; marked in filled circles of colours that are distinctive of the $X_{\mathrm{e}}$ value used - black for $X_{\mathrm{e}}=726 \mathrm{pc}$, red for $X_{\mathrm{e}}=1000 \mathrm{pc}$, green for $X_{\mathrm{e}}=1400$ pc and blue for $X_{\mathrm{e}}=2000 \mathrm{pc}$. These trends have been spotted during the parametric analysis that we undertake with our model galaxies. The recovered functional dependence that defines these trends are marked in solid lines of colour corresponding to the value of $X_{\mathrm{e}}$. These functional dependences were recovered using model galaxies with $\left\{n=3,5.3,6,7,8,9\right.$ all $\left.\mathrm{X}_{\mathrm{e}} \leq 2000 \mathrm{pc}\right\}$.

the profile in $w$ needs to be scaled. In fact, the value of $\Upsilon_{\text {out }}$ corresponding to observations in $w$ should be scaled by a factor of $10^{0.4\left(L_{\odot w}-L_{\odot z}\right)}$. Here $L_{\odot w}$ is the solar absolute magnitude in the $w$ band. In the ACS $z$-band $L_{\odot z}=4.52$. However, this transformation into the $w$-band is really an approximation since we assume all the way that $X_{\mathrm{e}}$ is the same over wavebands. This is not true (Temi et al. 2008; Ko \& Im 2005).

\subsection{Distance to the galaxy}

It is important to enquire about the stability of the posited forms of $\Upsilon_{\text {out }}$ and $\Upsilon_{\text {in }}$ when the distance to a galaxy is different from what has been used in the models, namely $17 \mathrm{Mpc}$ (approximate distance to Virgo). The only influence of the distance $D$ (in Mpc) to the observed galaxy is in affecting the luminosity density distribution that is obtained be deprojecting the observed surface brightness profile, through a term that is linear in $D^{-1}$. It is this luminosity density profile that is scaled by $\Upsilon_{\text {in }}$ and $\Upsilon_{\text {out }}$ in the inner and outer parts of the galaxy. Thus, if the galaxy is in reality further than $17 \mathrm{Mpc}$, the implementation of the suggested $M / L$ values would amount to an overestimation of the luminosity density. To compensate for this, we need to modulate the relevant $\Upsilon_{\text {out }}$ and $\Upsilon_{\text {in }}$ values by the factor $D / 17$.

\section{Tests}

In this section we discuss the testing of our advanced parametric forms of $\Upsilon_{\text {out }}=f\left(n, X_{\mathrm{e}}, \Upsilon_{\mathrm{in}}\right)$ (Eq. (9) with inputs from 10,11 and 12) and $\Upsilon_{\text {in }}^{\max }=g\left(n, X_{\mathrm{e}}, \alpha\right)$ (Eq. (8)). The scheme is tested on models that were excluded from the fitting exercise that resulted in our identification of the forms of the functions $f$ and $g$.

Figure 5 shows a comparison of the predicted and model mass density profiles along the $\boldsymbol{x}$-axis for the model galaxy that has a Sersic index of 3.5 and $X_{\mathrm{e}}=3000$ pc. $\alpha$ is set to $5 \Longrightarrow \sigma_{0} \approx 250 \mathrm{k} \mathrm{ms}^{-1}$, averaged over a radius of about $X_{\mathrm{e}} / 8=375$ pc. For this system, we extract $\Upsilon_{\text {in }}^{\max } \approx 5.8$ and $\Upsilon_{\text {out }} \approx 0.5$. This pair of $\Upsilon_{\text {out }}-\Upsilon_{\text {in }}$ values are used to scale the luminosity density distribution for this model galaxy which is then smoothed to offer a final mass density distribution along the major axis. These $\Upsilon_{\text {out }}-\Upsilon_{\text {in }}$ values imply a predicted mass density distribution (in red in Fig. 5) that tallies favourably with the model (in green in Fig. 5).

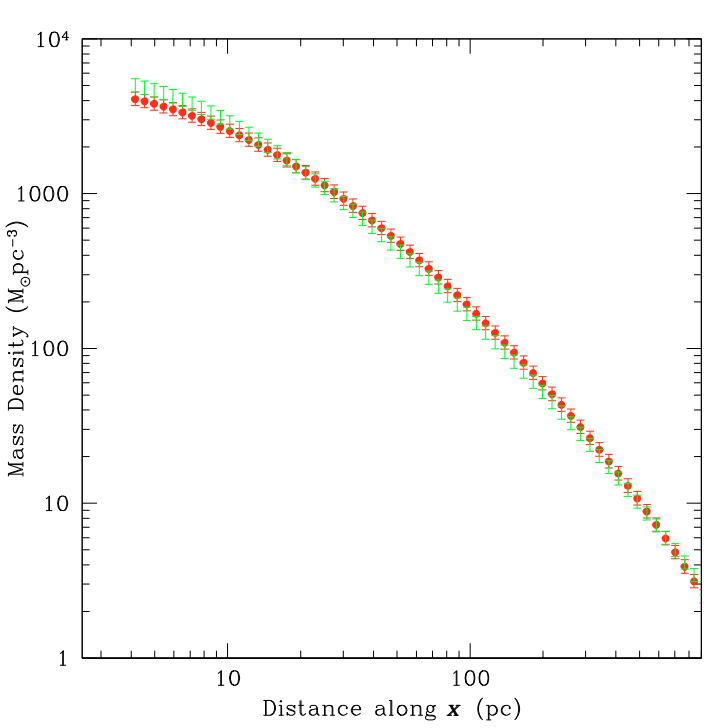

Fig. 5. Figure to compare the predicted total mass density distribution with $x$ (in red) with the known (model) mass density profile (in green), for the model described by $n=3.5$ and $X_{\mathrm{e}}=3000 \mathrm{pc}$. For an $\alpha$ of 5 , this model yields $\Upsilon_{\text {in }}^{\max } \approx 5.8$ and $\Upsilon_{\text {out }} \approx 0.5$. The profile in red results on scaling the luminosity profile of this model galaxy by the raw $M / L$ distribution that is defined by these "inner" and "outer" amplitudes and subsequently smoothing the discontinuous mass density profile thus obtained, (using the smoothing prescription mentioned in the text).

The predicted mass density distribution when compared to the luminosity density distribution offers the final or smoothed $M / L$ which is shown in Fig. 6 to be significantly different from the raw $M / L$ profile (in this black line).

However, this was the example of one given model galaxy, characterised by a given central velocity dispersion. Tests were undertaken to validate the relations predicted between the properties of the raw $M / L$ and the photometric parameters of model galaxies, across the full range of $\sigma_{0}$. The tests were carried out with model galaxies with Sersic index of 4 and $X_{\mathrm{e}}$ of $726 \mathrm{pc}$ and 1400 pc. In Fig. 7, we see the calculated $\Upsilon_{\text {out }}$ value corresponding to a given $\Upsilon_{\text {in }}$, for which compatibility is noted between the known (model) and predicted total mass density distributions, to 


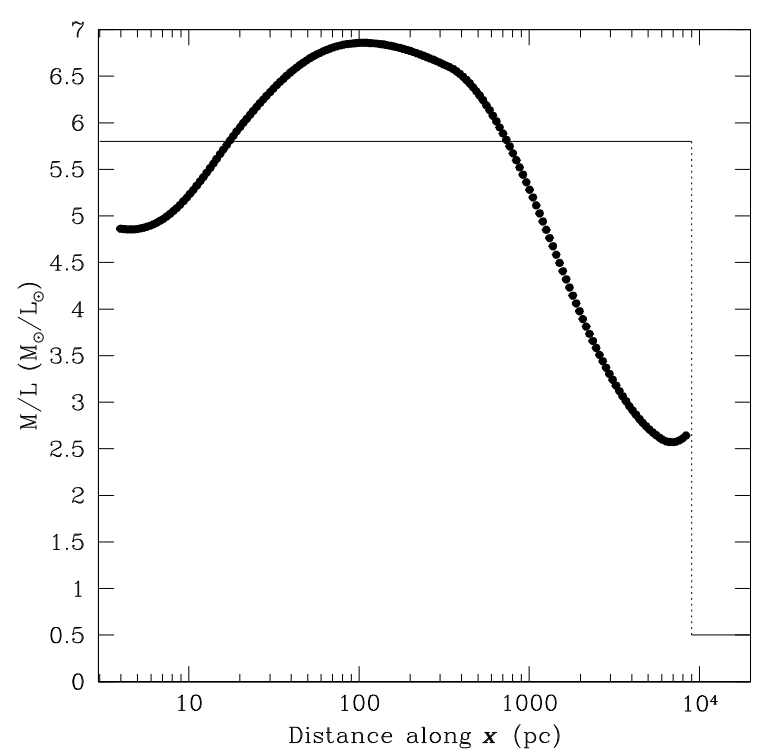

Fig. 6. Figure to compare the final total (local) $M / L$ distribution with the initially chosen raw $M / L$ profile (in broken lines), for the test galaxy the total mass of which is presented in Fig. 5. The final $M / L$ is presented within $3 X_{\mathrm{e}}$. The raw $M / L$ profile is the same as that shown in Fig. 1; as is apparent from this figure, it is significantly different from the final $M / L$ profile.

$3 X_{\mathrm{e}}$. Such $\Upsilon_{\text {out }}$ values are shown in the two panels of this figure, in black dots.

It is to be noted that these calculated values of $\Upsilon_{\text {out }}$ very closely straddle (within errors of $\pm 5 \%$ ) the analytical relationship between $\Upsilon_{\text {out }}$ and $\Upsilon_{\text {in }}$ that is predicted in Eqs. (9)-(12), for given $X_{\mathrm{e}}$ and $n$; in this case for $n=4, X_{\mathrm{e}}=726$ pc (left) and $n=4, X_{\mathrm{e}}=1400 \mathrm{pc}$ (right). Thus, these tests offer confidence in the formalism that we suggest.

\section{Applications}

In this section, we check out the efficacy of the advanced scheme in recovering gravitational mass density distribution of two elliptical galaxies, NGC 4494 and NGC 3379, to $3 X_{\mathrm{e}}$. Our predicted mass distributions are compared to independent dynamical mass models for these systems.

\subsection{NGC 3379}

NGC 3379 was reported by Romanowsky et al. (2004) and Douglas et al. (2007) to contain very little dark matter on the basis of a Jeans equation analysis of the kinematics of around 200 planetary nebulae $(\mathrm{PNe})$ that reside in the dark halo of this galaxy. An independent estimation of the distribution of the total mass density of this galaxy was performed by Chakrabarty (2009, submitted to AJ), by implementing these PNe velocities in the Bayesian algorithm CHASSIS (Chakrabarty \& Saha 2001; Chakrabarty \& Portegies Zwart 2005). As acknowledged by Chakrabarty (2009), these mass distributions from CHASSIS indicate somewhat higher masses than the estimates of Douglas et al. (2007), owing to the assumption of isotropy within CHASSIS. While details of such mass estimation techniques are irrelevant to the current work, here we present a comparison between the mass density profile obtained from our formalism with the same obtained from CHASSIS. We also present a comparison between the cumulative mass result $M(r)$ via the quantity defined as $v_{\mathrm{c}}=\sqrt{G M(r) / r}$, where $v_{\mathrm{c}}$ is referred to as the circular velocity.

Our predictions are based on the analysis of the photometry of NGC 3379 in the $B$-band (Capaccioli et al. 1990) and the data for projected central velocity dispersion, as given by Statler \& Smecker-Hane (1999). Using this $\sigma_{\mathrm{p}}$ data along the major axis of the galaxy, (Table $1 \mathrm{~A}$ of Statler \& Smecker-Hane 1999), we get an $\alpha$ of about 7.5. The photometry suggests an $X_{\mathrm{e}}$ of $2.2 \mathrm{kpc}$. Additionally, we deproject the $B$-band surface brightness profile using the Bayesian deprojection algorithm DOPING (Chakrabarty \& Ferraese 2008). We consider NGC 3379 to be at a distance of $11 \mathrm{kpc}$, as in Douglas et al. (2007). Our predicted values of (upper limit on) $\Upsilon_{\text {in }}$ and $\Upsilon_{\text {out }}$, as modulated by differences in the wave-band of the available photometry and distance to the system, are about 9.3 and 5.9.

The resulting mass density distribution that we advance for NGC 3379 within $3 X_{\mathrm{e}}$, is depicted in black on the left of Fig. 8. This is compared to the mass model identified by CHASSIS. $v_{\mathrm{c}}$ derived from our calculated mass distribution, from the dynamical mass modelling by CHASSIS and that reported by Douglas et al. (2007) are represented in the right panel of Fig. 8.

The comparison of the total mass density profile indicates the clear trend for our predicted mass distribution to be on the lower side compared to the gravitational matter density provided by CHASSIS. This is only to be expected since CHASSIS in its current form, assumes isotropy, which affects CHASSIS idiosyncratically to spuriously enhance mass density, as acknowledged by Chakrabarty (2009). Consequently, the $v_{\mathrm{c}}$ profile advanced by our work is also on the lower side of the profile that follows from CHASSIS, though our result compares better with the result advanced by Douglas et al. (2007).

\subsection{NGC 4494}

NGC 4494 is a nearby elliptical, the mass distribution of which to 7 effective radii has recently been presented by Napolitano et al. (2008). The distance to this galaxy is given as $15.8 \mathrm{Mpc}$ by Napolitano et al. (2008). The $V$-band photometry of this galaxy is presented in Table A1 of Napolitano et al. (2008). This surface brightness profile indicates an $X_{\mathrm{e}}$ of $3.37 \mathrm{kpc}$ and is deprojected, given the radial variation of the projected eccentricity (assuming an oblate geometry and edge-on viewing). The projection of such recovered luminosity density distribution is shown in the left panel of Fig. 9, compared to the surface brightness data of this galaxy (in red). Napolitano et al. (2008) also cite the central velocity dispersion of this system as $150 \mathrm{~km} \mathrm{~s}^{-1}$ (Paturel et al. 2003). Considering this $\sigma_{\mathrm{p}}$ to be the dispersion averaged over $R_{\mathrm{e}} / 8$, where $R_{\mathrm{e}}=48^{\prime \prime} .2$ for this galaxy, we obtain an $\alpha$ of 2 . Our predicted values of $\Upsilon_{\text {in }}$ and $\Upsilon_{\text {out }}$ are about 2 and 0.121 . These are used to characterise the raw $M / L$, which when smoothed, gives rise to the total mass distribution that is shown in the middle panel of Fig. 9.

The total $M / L$ ratio of NGC 4494, in the $V$-band is shown on the right panel of Fig. 9 while the enclosed gravitational mass distribution is depicted in the middle panel. This is very similar to the radial mass distribution of NGC 4494, within the inner $3 X_{\mathrm{e}}$, as reported by Napolitano et al. (2008, their Fig. 13).

\section{Discussions and summary}

We have presented a novel mechanism for estimating total mass density profiles of elliptical galaxies that can be described by 

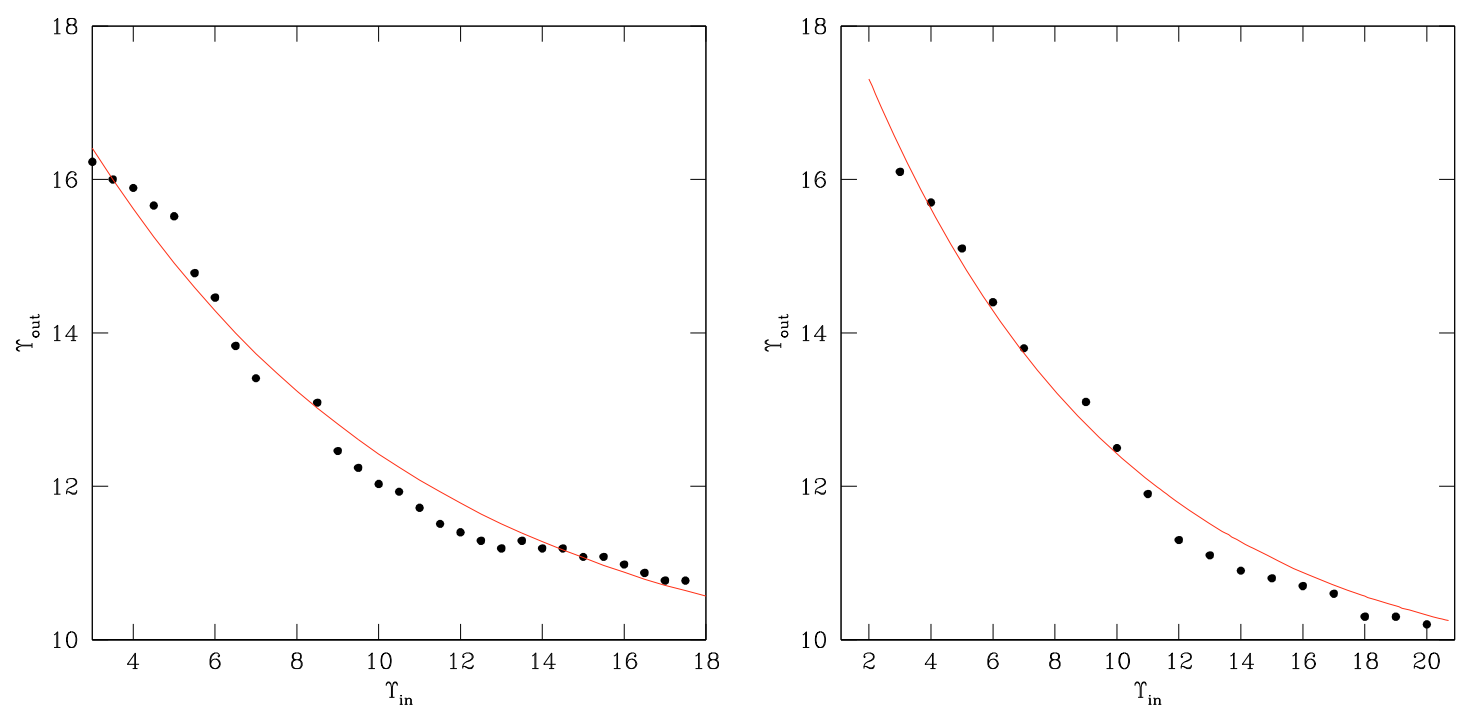

Fig. 7. Comparison of model $\Upsilon_{\text {out }}$, as obtained from our calculations (black filed circles) and predicted $\Upsilon_{\text {out }}$ (red line), for given $\Upsilon_{\text {in }}$, corresponding to model systems with Sersic index $=4$ and $X_{\mathrm{e}}=726 \mathrm{pc}($ left $)$ and $X_{\mathrm{e}}=1400 \mathrm{pc}($ right $)$. The predicted curves are obtained by using the values of $n$ and $X_{\mathrm{e}}$ for the model test system, in Eqs. (9)-(12). The calculated $\Upsilon_{\text {out }}$ values result from our analysis.
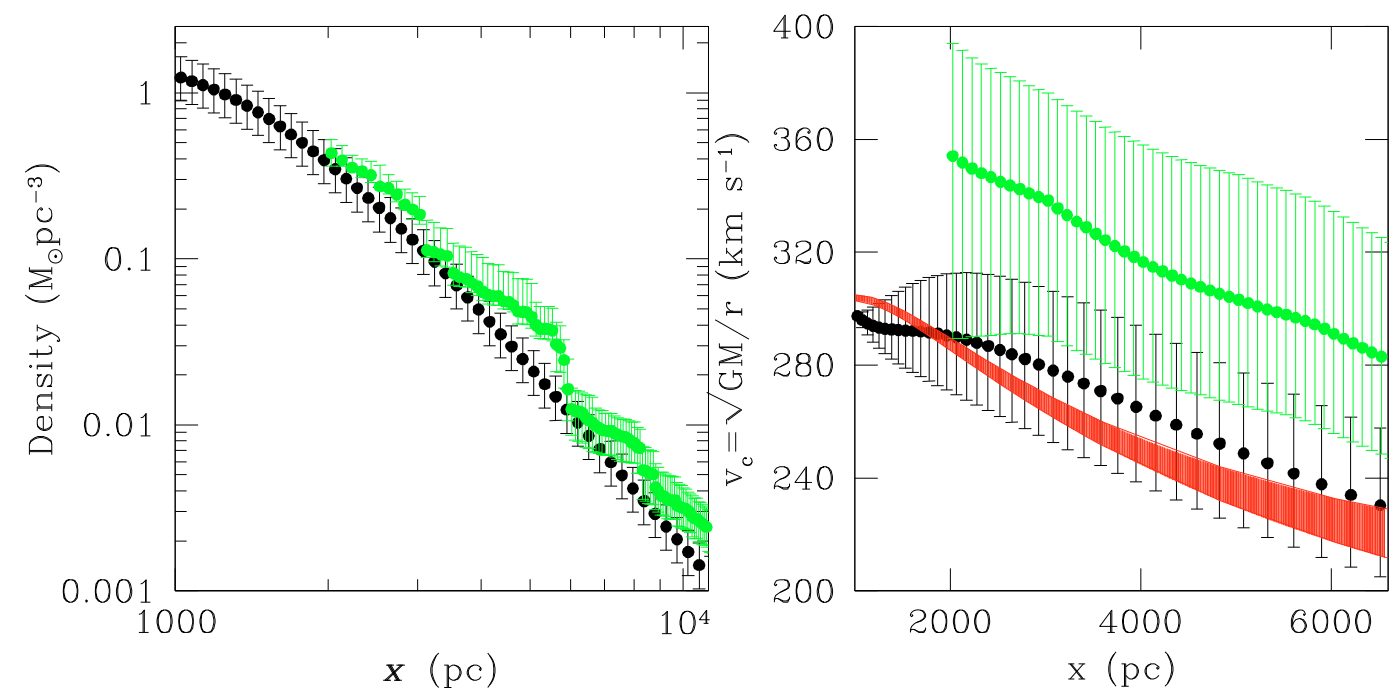

Fig. 8. The radial distribution of the recovered total mass density is depicted in black, on the left. On this is superimposed the mass density profile obtained by implementing the kinematic information of a sample of planetary nebulae in NGC 3379 (the same sample as used by Douglas et al. 2007), in the Bayesian algorithm CHASSIS (Chakrabarty 2009, communicated to AJ). The quantity $v_{\mathrm{c}}$, as recovered from these mass distributions are shown in corresponding colours on the right, with the $v_{\mathrm{c}}$ profile from Douglas et al. (2007) overplotted in red.

a Sersic-type surface brightness distribution. This formalism uses nothing in excess of what is typically available in the observational domain, namely, photometry and the central velocity dispersion profile. This allows the implementation of this scheme even for systems at high redshifts. (Such an implementation of this scheme will be presented in a future contribution - Chakrabarty \& Conselice, under preparation). The advanced scheme uses the methodology presented in Paper I, in the context of Sersic galaxies.

To begin with, we generate a sample of luminosity density distributions that project to Sersic brightness profiles, of assorted values of $n$ and $X_{\mathrm{e}}$. These toy galaxies are then assigned various values of the local central mass-to-light ratio (parametrised by $\alpha$ ). For each such configuration, we monitor the allowed range of $\Upsilon_{\text {in }}$ and the value of $\Upsilon_{\text {out }}$ that corresponds to any such $\Upsilon_{\text {in }}$. This pair of $\Upsilon_{\text {in }}-\Upsilon_{\text {out }}$ values define the raw or unsmoothed two- stepped local mass-to-light ratio distribution with $x$. The local mass density distribution in the system (to $3 X_{\mathrm{e}}$ ) is judged by scaling the luminosity density profile by the raw $M / L$ profile and then smoothing the result by the prescribed smoothing routine (two successive applications of a box filter of size corresponding to $X_{\mathrm{e}}$ ).

The dependence of $\Upsilon_{\text {out }}$ on $\Upsilon_{\text {in }}$ is found to be well approximated with an exponential decline that is described by three free parameters, which are actually all functions of the model characteristics, namely, $n$ and $X_{\mathrm{e}}$. The dependence of these three parameters on $n$ and $X_{\mathrm{e}}$ are extracted from our model-based study and analytical fits to these trends are presented. Such fits are noted to be favourably represented by polynomials.

The reliance of the band of the allowed values of $\Upsilon_{\text {in }}$ on $\alpha$ is also quantified as ranging from $\alpha$ to $\Upsilon_{\text {in }}^{\max }$, where the form of the function $\Upsilon_{\text {in }}^{\max }\left(n, X_{\mathrm{e}}, \alpha\right)$ is also found to be polynomial in nature. 

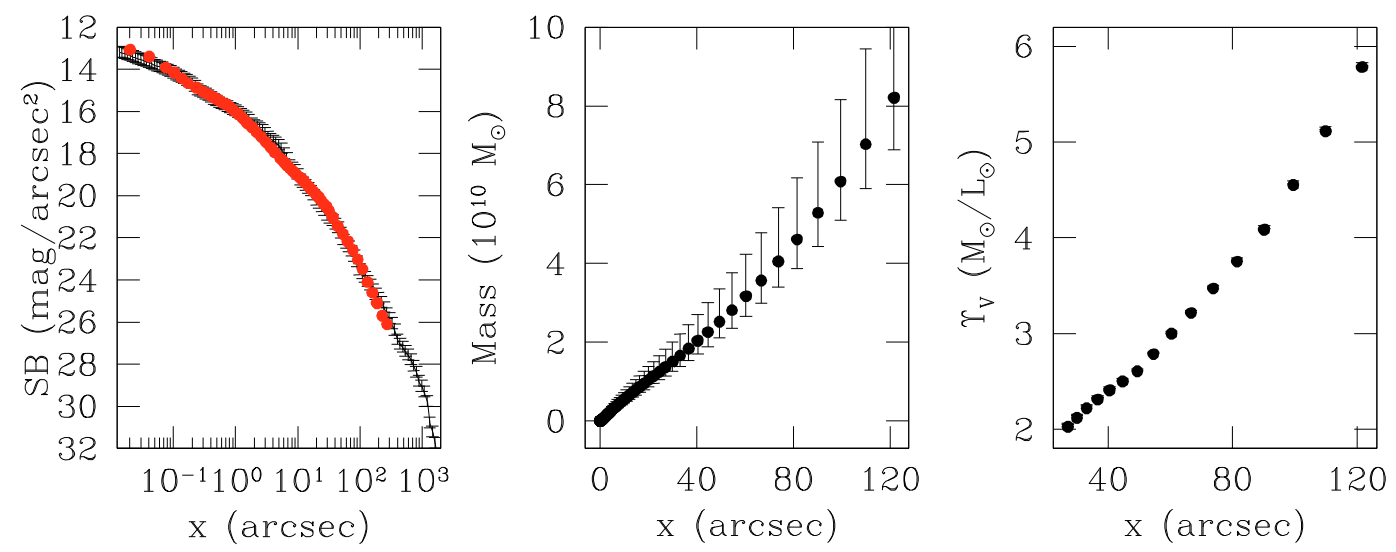

Fig. 9. Figure to bring out the results for the galaxy NGC 4494. The left panel depicts a projection of the luminosity density distribution that we estimate for the galaxy (in black), compared to the $V$-band surface brightness data (in red). The total gravitational mass distribution of the galaxy is represented in the middle panel while the mass-to-light ratio on the $V$-band is shown on the right.

\subsection{Deviation from predicted trends?}

Even though the polynomial fits are found to do a good job for most configurations, for certain models, the predictions appear incorrect (by no more than $10 \%$ ), e.g. $\tau$ may be judged underestimated for $n=8$ and 9 for the configuration: $X_{\mathrm{e}}=2000 \mathrm{pc}$. However, this should be interpreted as erroneous recording of $\Upsilon_{\text {out }}$ for these $\Upsilon_{\text {in }}$ values from our models, rather than a failure of our predictions. Typically, such errors emanate from lack of refinement in the step-sizes used in our work and is potentially amendable.

\subsection{Applicable to. .}

As delineated in Sect. 3, the formalism that we advance here, is applicable only to galaxies with Sersic indices $n \geq 3$. It is only for such systems that the luminosity density profile is steep enough, i.e. falls quickly enough to ensure that in the "outer" parts of the system the luminous matter density is completely overwhelmed by the dark matter density, so that the total matter density is effectively a representation of the dark matter distribution. Thus, changes in the central $M / L$ or $\alpha$ do not affect the $M / L$ in these "outer" parts. Now, to qualify "outer", we state that this region corresponds to $x \sim 3 X_{\mathrm{e}}$. This is anyway true, only if the luminous matter fraction is not too small, in which case, we need to settle for progressively lower $M / L$ in the outer parts, as $\alpha$ increases.

\subsubsection{Effect of lowering Sersic Index}

If however, the luminous density profile is too flat, (as for $n<$ 3 ), the contribution of the luminous matter, to the total mass, is significant, even in "outer" parts. In order to achieve similarly shaped total mass profiles, the value of $\Upsilon_{\text {out }}$ will need to increase with increasing $\alpha$. Then, the exponential fall-off of $\Upsilon_{\text {out }}$ with $\Upsilon_{\text {in }}$ will not be noted. Qualitatively speaking, this is the reason why our formalism will not be valid for galaxies with Sersic indices less than 3.

\subsubsection{Effect of increasing size}

However, this increased flattening of the luminosity density profile can be attained even for $n \geq 3$, for large $X_{\mathrm{e}}$ values. Thus, in such systems, for the same reason as in the last paragraph, $\Upsilon_{\text {out }}$ will increase with an increase in the inner $M / L$ instead of falling exponentially with increasing $\Upsilon_{\text {in }}$. Thus, based on these considerations, on the basis of our experiments, we find that the following galaxies cannot be accommodated within our suggested formalism:

$$
\begin{aligned}
& -n=3 \text { and } X_{\mathrm{e}}>4000 \mathrm{pc} \\
& -n=4 \text { and } X_{\mathrm{e}}>5000 \mathrm{pc} \\
& -n<3
\end{aligned}
$$

\subsubsection{What the ranges mean}

However, it is important to keep in mind that these ranges have been ascertained on the basis of a discretised scanning of the parameters $n$ and $X_{\mathrm{e}}$, of our two-parameter galaxy models. Thus, when we say that models with $n<3$ cannot be explained with the predicted relationships between $\alpha, \Upsilon_{\text {in }}, \Upsilon_{\text {out }}$, what we really mean is that our experiments with a model with $n=2$ indicated failure of adherence to these predicted trends. It is very much possible that a model with an intermediate Sersic index can be explained by our predictions, such as a galaxy with $n=2.3$, say. In other words, the ranges of $n$ and $X_{\mathrm{e}}$ described above owes, to our dealing only with models ascribed the aforementioned discrete values (values mentioned in Sect. 3). Consequently, the ranges listed above are a conservative interpretation.

The practical question to ask is, are these suggested ranges compatible with real galaxies? As itemised in Sect. 3, our choice is supported by the trends observed for the Virgo cluster, within the ACS Virgo Cluster Survey, in alliance with the predicted evolution towards greater compactness in high-redshift systems (Ferreras et al. 2009). Furthermore, keeping in mind that a waveband dependence of effective radius exists, our model $X_{\mathrm{e}}$ values in the $z$-band fall within limits indicated by observations (Ferrarese et al. 2006; Trujillo et al. 2001; La Barbera et al. 2005).

Additionally, we question the relevance of the upper limits on the effective radii at $n=3$ and 4 , which are smaller than the range permitted for higher values of $n$. As far as the correlation between the shape parameter $n$ and $\log$ of effective radius is concerned, it is well known that in general, half-light radius decreases with decreasing $n$ (Boselli et al. 2008; Côté et al. 2008; Naab \& Trujillo 2006; Brown et al. 2003; Trujillo et al. 2001). In fact, such a correlation is physical and is hinted at by the global relations between structural parameters; it is not merely a reflection of the fitting procedure (Trujillo et al. 2001). 

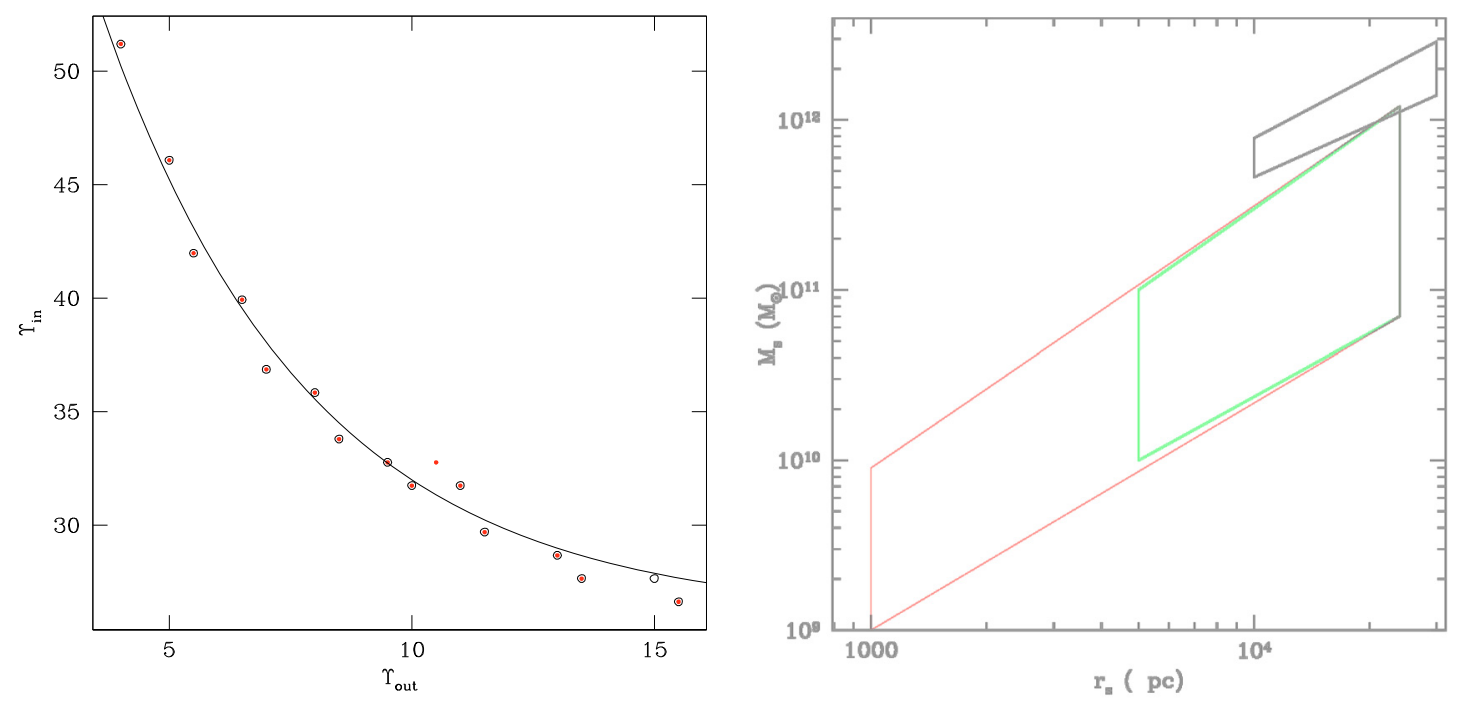

Fig. 10. The left panel depicts the relation between $\Upsilon_{\text {out }}$ and $\Upsilon_{\text {in }}$, obtained using the model with $n=5.3$ and $X_{\mathrm{e}}=1000$ pc, from two different runs that seek this relation, given NFW haloes characterised by $\left(M_{\mathrm{s}}, r_{\mathrm{s}}\right)$ values within the quadrilaterals in black (see right panel) and in red (see right panel). When the halo density parameters are as contained within the black quadrilateral, the obtained $\Upsilon_{\text {out }}-\Upsilon_{\text {in }}$ relation is shown in open black circles in the left panel. When the halo density is characterised by $M_{\mathrm{s}}$ and $r_{\mathrm{s}}$ values as points inside the red quadrilateral on the right, the $\Upsilon_{\text {out }}-\Upsilon_{\text {in }}$ relation is shown in red filled circles in the left panel. The predicted $\Upsilon_{\text {out }}-\Upsilon_{\text {in }}$ relation in shown on the left in solid black lines. The green quadrilateral represents the subset in the $M_{\mathrm{s}}-r_{\mathrm{s}}$ space, which defines the dark matter density distributions for which concurrent $f$ and $g$ functions were recovered in general runs done with assorted models, within our scheme.

\subsubsection{Effect of model dark matter distribution}

Of course, it could be perceived that such fate of the mass distribution at $x \sim 3 X_{\mathrm{e}}$ would be dictated by the exact details of the underlying dark matter distribution that defines the model galaxy. Indeed, for the high values of $n$, the $M / L$ at $x \geq 3 X_{\mathrm{e}}$ $\left(\Upsilon_{\text {out }}\right)$ is dictated by the details of the dark matter distribution, but it is the smoothing of this raw $M / L$ profile that gives us the end product, namely the total local $M / L$ ratio profile of the galaxy upto the benchmark $x$ of $3 X_{\mathrm{e}}$. It is precisely this smoothing procedure that brings into the final $M / L$ structure, elements of the mass distribution in the inner $3 X_{\mathrm{e}}$ in the galaxy, as well as a relatively less significant signature of the same that lies outside the radius upto which the distribution is sought. The difference that smoothing makes to the raw $M / L$ profile, as distinguished from the final $M / L$ profile, is clear in Fig. 6.

Thus, given such (1) implementation of our smoothing procedure and that (2) the jump radius in the raw $M / L$ profile is itself $3 X_{\mathrm{e}}$, we find that our experiments bear the fact that changing the mass scale and length scale of the used NFW dark matter density distribution across the wide ranges do not affect the mass configuration for $x \lessgtr 3 X_{\mathrm{e}}$, though further out in the galaxy, the influence of changing the DM density distribution picks up quickly. The consistency in the $\Upsilon_{\text {out }}-\Upsilon_{\text {in }}$ relation, noted with changes in $M_{\mathrm{s}}$ and $r_{\mathrm{s}}$ is brought out in the left panel of Fig. 10. The allowed ranges in $M_{\mathrm{s}}$ and $r_{\mathrm{s}}$ are provided in the right panel of Fig. 10.

\subsubsection{Why choose NFW?}

The modelling of the dark matter distribution in early type galaxies is obviously indirect and consequently difficult and unreliable. Ferreras et al. (2008) suggest a large scatter in the outer slope of the dark matter distributions that they recover for their targeted galaxies. Gavazzi et al. (2007) corroborate an NFW modelling of the dark haloes of their sample galaxies, suggesting that the total matter density tends to an overall isothermal form. Given this degree of uncertainty, it might be argued that there is not much sense in splitting hair to decide between an NFW and isothermal dark matter distributions, as long as the dark haloes that we probe in our analysis are compatible with observations or simulations.

This is indeed the case, when we compare our ranges of halo characteristics (right panel of Fig. 10) to the suggestion by Kleinheinrich et al. (2006) that the recovered range of virial masses of their NFW model to be $\left[3.9 \times 10^{11}, 7.1 \times 10^{11}\right] M_{\odot}$. Such a mass range is compatible with the suggestion by Hoekstra et al. (2004) for a fiduciary galaxy of luminosity $L_{B}=10^{10} L_{B \odot}$ that has an NFW profile $\left(M_{200} \in[10,6.4] \times 10^{11} M_{\odot}\right.$, as indicated by Fig. 4 in Hoekstra et al. 2004). This mass range corresponds to a range of about $8 \mathrm{kpc}$ to about $30 \mathrm{kpc}$ for $r_{\mathrm{s}}$. In fact, we cover these ranges in the runs, the results of which are presented in Fig. 10, and also scan haloes of lower masses. For various haloes defined within the red and black quadrilaterals in Fig. 10, compatible $\Upsilon_{\text {out }}-\Upsilon_{\text {in }}$ relations were recovered.

This explains our choice of sticking with the NFW prescription for our dark halo model. For reasons similar to what we explain above, we suggest that upon smoothing, haloes of varying shapes but similar masses within $3 X_{\mathrm{e}}$ are not expected to affect the mass distribution within $3 X_{\mathrm{e}}$ in the massive ellipticals that we deal with in this formalism.

Thus, we see that our methodology is valid, irrespective of selecting haloes defined by widely different $M_{\mathrm{S}}$ and $r_{\mathrm{s}}$ values, (i.e. NFW haloes defined by points inside the black and red quadrilaterals in Fig. 10). This motivates us to choose to work with a smaller subset of all the haloes explored in these runs; in particular, we choose to work with haloes defined by the more massive half of the $\log \left(M_{\mathrm{s}}\right)$ range that limits the red quadrilateral. This chosen subset in the $M_{\mathrm{s}}-r_{\mathrm{s}}$ space is bound by the green quadrilateral in Fig. 10. 


\subsubsection{Effect of model geometry}

In addition to details of the DM distribution, we admit that the model structure does include the free parameters that describe the Sersic model of density distributions in general triaxial galaxies, as it ideally should, though this work has full potential of being extended into two-dimensions and include an axisymmetric description of the galaxy. Now, the triaxiality of the models needs to be defined in terms of a chosen geometry and intrinsic eccentricities - it is of course not possible to constrain such characteristics from observations alone.

In our models, these were fixed as oblateness with an axial ratio of 0.7 . It is indeed possible that the unknown functions $f$ and $g$ that we attempt to constrain, harbour dependence on these intrinsic geometric factors. However, the preparatory assumptions involved in the deprojection of an observed brightness distribution, namely the underpinning of intrinsic geometry and viewing angle, are essentially unconstrained, unless the system is favourably inclined or flattened. In other words, the unknown in our modelling are the usual quantities that render deprojection non-unique.

As for the specification of the ellipticity in our models, a typical value has been adopted - Padilla \& Strauss (2008) suggest an axial ratio distribution for a large sample of SDSS ellipticals, with a mode in the range of 0.6 to 0.8 . Inspired by this, we use an axial ratio of 0.7 in our models. Again, the uncertainty in intrinsic ellipticity, cannot be known for a general observed galaxy. The quantification of the deprojection effects on the formulae provided above is possible, at least in a statistical sense, and it is envisaged that the same will be pursued in the future.

\subsection{What if central super massive black holes?}

The case of a central mass condensation in the system was dealt with in Paper I, in reference to the example of the galaxy M 87 the recovered mass distribution of M 87 was demonstrated to be consistent with the same obtained from kinematical considerations. If independent measurements indicate an observed system to harbour a massive central mass condensation, then the scheme delineated in Paper I will be followed.

\subsection{Why $X_{\mathrm{e}}$ instead of $R_{\mathrm{e}}$ ?}

The usage of $X_{\mathrm{e}}$ instead of $R_{\mathrm{e}}$ is preferred since $X_{\mathrm{e}}$ as per its definition here, as well as in Paper I, is derived solely from the inputs to the methodology, namely the surface brightness profile along the semi-major axis which we consider to be along the $\hat{\boldsymbol{x}}$-axis. Thus, any changes to the shape of the brightness profile will be directly reflected in a linear change in $X_{\mathrm{e}}$ but not necessarily so in the half-light radius that is estimated from isophotal analysis. In fact, here we use the same definition for $X_{\mathrm{e}}$ as in Paper I, (see Sect. 2).

The usage of $X_{\mathrm{e}}$ should not be cause for concern since $X_{\mathrm{e}}$ is merely one definition of the semi-major axis effective radius and reduces to the conventional definition of the major-axis effective radius for $n=4^{1}$. When an unknown galaxy is being analysed within this formalism, its half-light radius is just as much an unknown as is the $X_{\mathrm{e}}$ that we define here. Thus, there is no loss of connection with observations by the implementation of $X_{\mathrm{e}}$.

\footnotetext{
${ }^{1}$ Semi-major axis effective radius has been used before, for example by Naab \& Trujillo (2006).
}

\subsection{Effect of choice of smoothing prescription}

It merits mention that other choices for the smoothing prescription and $x_{\text {in }}$ may also work, but here we concentrate on the above mentioned configuration and the specification of $\Upsilon_{\text {in }}$ and $\Upsilon_{\text {out }}$ are accordingly unique to these choices.

\subsection{Effect of choice of estimation of central local $M / L$}

In a similar context, it may be argued that the definition of $\alpha$ that we use herein will leave an imprint. We equate $\alpha$ to the central local $M / L$, inspired by this result that is achieved in Paper I. Here the total gravitational mass found enclosed within the radius $x_{0}$ is $M\left(x_{0}\right)$ where $M\left(x_{0}\right)$ is linked to the $3-\mathrm{D}$ velocity dispersion at $x_{0}$ via: $\sigma_{0}^{2}=G M\left(x_{0}\right) / x_{0}$. Then, according to our definition, $\alpha$ is given as the ratio of $M\left(x_{0}\right)$ and the enclosed light within $x_{0}$. For other definitions of $\alpha$, other ranges of $\Upsilon_{\text {in }}$ will be valid (and therefore other forms of dependences of $\Upsilon_{\text {out }}$ on $\Upsilon_{\text {in }}$, for the same galaxy). Thus, the pairs of $f$ and $g$ functions that we advance here, work for the used choice of the definition.

We would like to emphasise that the extraction of the exact value of the mass enclosed within $x_{0}$ (and therefore of $\alpha$ ), from a measurement of $\sigma_{0}$, is not the point of this exercise; uncertainties in this extraction do not undermine the advanced results either, as long as the galaxy at hand is "not too" aspherical or anisotropic at $x_{0}$. Here we qualify "not too" as those configurations for which we obtain consistent mass profiles using the two extreme values of $\Upsilon_{i n}$, that are allowed for the extracted value of $\alpha$. Thus, when the method fails, we know that it does. As long as this aforementioned consistency is noted, choosing $\Upsilon_{\text {in }}$ from anywhere within the range corresponding to the given $\alpha$ will lead to consistent total mass distributions within $3 X_{\mathrm{e}}$. Additionally, this range is neither too constricted nor too relaxed, as was discussed in Paper I.

\subsection{Comparatively better applicability}

The presented device is based upon conclusions that are drawn from a sample of model Sersic galaxies. This would naturally imply that the success of this formalism is crucially dependant on the generality of the models. In particular, we have discussed the ranges of $n$ and $X_{\mathrm{e}}$ for which our advanced results are true. We have also discussed the effect of changing properties of the dark halo that we use in the models and find the advanced scheme robust to such model parameter variations.

In fact, the formalism presented above is unique in its scope and structure. Estimates of total mass distributions in distant elliptical systems are difficult and therefore rare; the formulae presented herein are therefore advantageous and could be treated as guides to decipher the total mass distribution of Sersic galaxies in large surveys.

Most importantly, the advanced methodology is successful within a severely constricted data domain, compared to any other scheme that aims to obtain mass distributions in elliptical galaxies. All that the advanced method demands in terms of data is what is typically available - surface brightness profile and a measure of central velocity dispersion. The undemanding nature of our method renders it applicable even at high redshifts. The simplicity of implementation of the input data is advantageous in that it allows for the scheme to be used in an automated way, to obtain mass distributions for large samples of galaxies. Tricks such as this and Nipoti et al. (2008) exploit the basic configuration within galaxies and offer novel ways for characterisation of distant systems. 
Acknowledgements. D.C. is funded by a Royal Society Dorothy Hodgkin Fellowship. B.J. acknowledges the support of a University of Nottingham Summer Studentship. We thank Sebastian Foucaud for useful discussions that helped enrich the paper.

\section{References}

Bolton, A. S., Treu, T., Koopmans, L. V. E., et al. 2008, ApJ, 684, 248 Boselli, A., Boissier, S., Cortese, L., \& Gavazzi, G. 2008, A\&A, 489, 1015 Brown, R. J. N., Forbes, D. A., Silva, D., et al. 2003, MNRAS, 341, 747

Buitrago, F., Trujillo, I., Conselice, C. J., et al. 2008, ApJ, 687, L61

Capaccioli, M., Held, E. V., Lorenz, H., \& Vietri, M. 1990, AJ, 99, 1813

Cappellari, M., et al. 2006, MNRAS, 366, 1126

Chakrabarty, D., \& Ferrarese, L. 2008, International Journal of Modern Physics D, as part of Proceedings for the 6th International Workshop on Data An alysis in Astronomy, Modelling and Simulations in Science, 17, 2

Chakrabarty, D. 2007, MNRAS, 377, 30

Chakrabarty, D., \& Portegies Zwart, S. 2004, ApJ, 128, 1046

Chakrabarty, D., \& Saha, P. 2001, AJ, 557, 292

Churazov, E., Forman, W., Vikhlinin, A., et al. 2008, MNRAS, 388, 1062

Côté, P., et al. 2008, IAU Symp., 245, 395

Czoske, O., Barnabè, M., Koopmans, L. V. E., Treu, T., \& Bolton, A. S. 2008, MNRAS, 384, 987

de Lorenzi, F., Gerhard, O., Saglia, R. P., et al. 2008, MNRAS, 385, 1729

de Lorenzi, F., Debattista, V. P., Gerhard, O., \& Sambhus, N. 2007, MNRAS, 376, 71

Diehl, S., \& Statler, T. S. 2007, ApJ, 668, 150

Douglas, N. G., Napolitano, N. R., Romanowsky, A. J., et al. 2007, ApJ, 664, 257

Ferrarese, L., Côté, P., Blakeslee, J. P., et al. 2006, [arXiv : astro-ph/0612139]

Ferreras, I., Lisker, T., Pasquali, A., Khochfar, S., \& Kaviraj, S. 2009, [arXiv: 0901.4555]

Ferreras, I., Saha, P., Williams, L. L. R., \& Burles, S. 2008, IAU Symp., 244, 206

Mahdavi, A., Hoekstra, H., Babul, A., \& Henry, J. P. 2008, MNRAS, 384, 1567
Fukazawa, Y., Botoya-Nonesa, J. G., Pu, J., Ohto, A., \& Kawano, N. 2006, ApJ, 636, 698

Gavazzi, R., Treu, T., Rhodes, J. D., et al. 2007, ApJ, 667, 176

Hoekstra, H., Yee, H. K. C., \& Gladders, M. D. 2004, ApJ, 606, 67

Humphrey, P. J., Buote, D. A., Brighenti, F., Gebhardt, K., \& Mathews, W. G. 2008, ApJ, 683, 161

Kleinheinrich, M., et al. 2006, A\&A, 455, 441

Ko, J., \& Im, M. 2005, J. Korean Astronomical Society, 38, 149

Koopmans, L. V. E., \& Treu, T. 2003, ApJ, 583, 606

La Barbera, F., Covone, G., Busarello, G., et al. 2005, MNRAS, 358, 1116

Lemze, D., Barkana, R., Broadhurst, T. J., \& Rephaeli, Y. 2008, MNRAS, 386, 1092

Lima Neto, G. B., Gerbal, D., \& Márquez, I. 1999, MNRAS, 309, 481

Mazure, A., \& Capelato, H. V. 2002, A\&A, 383, 384

Naab, T., \& Trujillo, I. 2006, MNRAS, 369, 625

Napolitano, N. R., Romanowsky, A. J., Coccato, L., et al. 2008, [arXiv:0810.1291]

Navarro, J. F., Frenk, C. S., \& White, S. D. M. 1996, ApJ, 462, 563

Nipoti, C., Treu, T., \& Bolton, A. S. 2008, MNRAS, 390, 349

O’Sullivan, E., \& Ponman, T. J. 2004, MNRAS, 354, 935

Padilla, N. D., \& Strauss, M. A. 2008, MNRAS, 388, 1321

Padmanabhan, N., Seljak, U., Strauss, M. A., et al. 2004, New Astron., 9, 329

Paturel, G., Petit, C., Prugniel, P., et al. 2003, A\&A, 412, 45

Prugniel, P., \& Simien, F. 1997, A\&A, 321, 111

Romanowsky, A. J., Douglas, N. G., Arnaboldi, M., et al. 2004, Science, 301, 1696

Sersic, J. L. 1968, Cordoba, Argentina: Observatorio Astronomico

Statler, T. S., \& Smecker-Hane, T. 1999, AJ, 117, 839

Temi, P., Brighenti, F., \& Mathews, W. G. 2008, ApJ, 672, 244

Terzić, B., \& Graham, A. W. 2005, MNRAS, 362, 197

Terzić, B., \& Sprague, B. J. 2007, MNRAS, 377, 855

Trujillo, I., Asensio Ramos, A., Rubiño-Martín, J. A., et al. 2002, MNRAS, 333, 510

Trujillo, I., Graham, A. W., \& Caon, N. 2001, MNRAS, 326, 869

Zhang, Z., Xu, H., Wang, Y., et al. 2007, ApJ, 656, 805 